\title{
The Focal Mechanical Vibration for Balance Improvement in Elderly - A Systematic Review
}

\author{
Teresa Paolucci' \\ Letizia Pezzi (D) \\ Roberta La Verde ${ }^{2}$ \\ Pasqualino Maietta Latessa ${ }^{3}$ \\ Rosa grazia Bellomo ${ }^{4}$ \\ Raoul Saggini ${ }^{\prime}$ \\ 'University G. d'Annunzio Chieti, \\ Department of Medical and Oral Sciences \\ and Biotechnologies, Chieti-Pescara, Italy; \\ ${ }^{2}$ S. Filippo Neri Hospital, Physical \\ Medicine and Rehabilitation, "Sapienza" \\ University, Rome, Italy; ${ }^{3}$ Department for \\ Life Quality Studies, University of \\ Bologna, Bologna, Italy; ${ }^{4}$ University of \\ Study of Urbino Carlo Bo, Department of \\ Biomolecular Sciences, Urbino, Italy
}

Correspondence: Teresa Paolucci G. D'Annunzio University of ChietiPescara, Rehabilitation Unit, Department of Medical and Oral Sciences and Biotechnologies (DSMOB), Via Dei Vestini 66100, Chieti Scalo, Italy

Email teresapaolucci@hotmail.com
Background: Aging has been associated with the progressive depletion of lean mass, reductions in muscle strength and the coordination of the lower extremities, accompanied by decreased gait assurance and balance control. Also, less balance control favors falling which is the leading cause of injury among the elderly. The aim of this systematic review is to identify and evaluate existing evidence regarding the use of focused vibration (FV) to improve balance and reduce the risk of falling during the rehabilitation of elderly populations.

Methods: The PICO question is what are the effects of focal/segmental/local vibration training on the assessment of balance and the risk of falls among the elderly population? A thorough literature review was conducted between May 1, 2009, and June 30, 2019, for studies in English, randomized clinical trials, including crossover and prospective design studies with assessing balance and the risk of falls in elderly populations (age $>60$ years).

Results: Eight articles $(\mathrm{N}=8)$ satisfied the inclusion criteria and were considered, of which 6 are RTC, one cross-sectional study and one clinical study, for a total of 635 participants. A total of 6 different vibration devices were used, each of which was associated with different FV frequency and amplitude characteristics and different treatment protocols.

Conclusion: In conclusion, FV can be effective in decreasing the risk of falls and improving the assessment of balance, but more evidence is necessary considering the limits of the studies; however, it does look an important promise during rehabilitative treatment.

Keywords: rehabilitation, risk of falls, exercise, vibration, equilibrium

\section{Introduction}

The progressive aging of the population is a phenomenon that can no longer be ignored. According to the World Health Organization (WHO), the proportion of people aged older than 60 years is increasing faster than other age groups, in all countries; for instance, in 2016, 19\% of the European population was aged 65 years or older, and this group is expected to encompass $30 \%$ of the population by $2060{ }^{1}$

Aging has been associated with the progressive depletion of lean mass, especially muscle mass, a phenomenon known as "sarcopenia" resulting in the loss of muscle strength, loss of mobility, neuromuscular impairments, and homeostatic balance failure syndrome, accompanied by gait and balance disorders. ${ }^{2}$ Sarcopenia is a multifactorial process, associated with intrinsic causes [decreased levels of growth hormone $(\mathrm{GH})$ and sex hormones and decreased numbers of motor neurons] and extrinsic or environmental causes (nutrition, partial or total immobility, and a lack of exercise). ${ }^{3,4}$ With advancing age, motor units, "i.e." an $\alpha$ motor neuron and the muscle fibers it innervates, become reduced, in total number and size, causing changes in the abilities of muscle tissue to generate strength. ${ }^{5}$ 
Muscle strength begins to decrease rapidly after the age 65 years, with the lower limb muscles, particularly the quadriceps femoris muscle being affected more than other muscle groups. ${ }^{6}$ These changes have negative effects on balance maintenance and posture among the elderly, which have been associated with reductions in autonomy and the establishment of a "fear of falling" as a serious consequence since it leads to a mobility reduction, social isolation, and diminished quality of life. ${ }^{6,7}$

In the present study, we have defined a fall to be an event that "results in a person coming to rest inadvertently on the ground or other lower level and other than as a consequence of a violent blow, loss of consciousness, or sudden onset of paralysis". ${ }^{8}$ Falls are the leading cause of injury among the elderly and have been associated with increased morbidity, functional decline, reductions in social activity, and poor quality of life. Further, many indicators of frailty, such as poor vision, low handgrip strength, decline in walking speed, the use of walking aids, drug use, and depression, have been recognized as risk factors for falls. ${ }^{9}$ To reduce the risk of falls, evidence in the literature supports a crucial role for exercise, especially exercises that are designed to improve balance and gait and increase lower limb strength. ${ }^{10}$

Rehabilitation plays a similarly important role as exercise, with the aim of addressing the disabilities that are characteristic among the elderly population. The primary aim of rehabilitation among the elderly is the recovery and maintenance of the bodily functions that are necessary to perform independent activities of daily life (ADLs). ${ }^{10}$

Among the elderly, the importance of physical exercise for maintaining muscle strength, optimizing reaction times, and improving balance and coordination has been demonstrated. ${ }^{11}$ Moreover, several studies have reported specific benefits in response to whole-body vibration training in the older population, including improvements in balance and gait speed. ${ }^{12}$ In fact, Sarabon et al, in a recent meta-analysis, showed that whole-body vibration training in the older population seems to be comparably effective for improving muscle strength, but not muscle cross-sectional area. ${ }^{13}$ However, limitations exist for the administration of whole-body vibration training, especially among the elderly population, who have numerous comorbidities, such as cardiac and vascular diseases. ${ }^{14}$ Several adverse effects following whole-body vibration exposure have been reported, including low back pain; circulatory disorders, such as Raynaud syndrome, nervous system alterations; perception disorders, and dizziness. However, most of these adverse effects have been reported following prolonged exposure to vibrations for occupational purposes. $^{15}$ Therefore, during rehabilitation procedures, focused vibration (FV) is generally preferred, which allows the stimulation of individual muscle groups and selectively activates type Ia and IIb fibers and the Golgi tendon organs, depending on the stimulus. ${ }^{16}$

In a recent review, Aboutorabi et $\mathrm{al}^{17}$ investigated the existing reports of the effects of FV interventions on postural control and gait among the elderly, particularly the use of vibratory insoles and the application of localized vibrations to the ankle and foot. Vibrating footwear appeared to improve balance [based on reductions in center of pressure $(\mathrm{CoP})$ velocity and displacement] in healthy elderly individuals and increased the walking speed, cadence, step time, and step length among stroke patients. If the exteroceptive afferents found on the sole of the foot play important roles during balance maintenance, then multiple pieces of sensory information must be evaluated by the central nervous system and integrated with other stimuli, to maintain an erect posture. ${ }^{18}$ Postural control strategies can include "reaction" (feed-back), "anticipation" (feed-forward), or a combination of both. Postural control is a complex motor skill that relies on interactions between multiple sensorimotor processes; thus, even the slightest age-related changes in the peripheral and central components of the visual, somatosensory, and vestibular systems can affect balance and mobility. ${ }^{19}$ With decreased cognitive functions, these impairments lead to an increased risk of falling among the elderly. ${ }^{11-20}$ Therefore, the aim of this systematic review was to identify and evaluate existing evidence regarding the use of FV to improve balance and reduce the risk of falling during the rehabilitation of elderly populations.

\section{Materials and Methods}

\section{Search Strategy}

The method for conducting this systematic review is based on the guidelines that have been established by the Preferred Reporting Items for Systematic Reviews and Meta-Analyses (PRISMA) statement. ${ }^{21}$

A systematic review of the literature was performed, using the following search engines: PubMed, PEDro, Scopus, and Cochrane Library. To perform the search, the following algorithm was developed, based on the PICO acronym, ${ }^{22}$ to evaluate a Population (elderly), Intervention (focal/segmental/local vibration training), 
Comparator (no treatment, placebo, or other exercises), and Outcomes (postural control, risk of falls, and functional balance). The research question was as follows: What are the effects of focal/segmental/local vibration training on the assessment of balance and the risk of falls among the elderly population?

The primary outcome of interest was the assessment of balance, whereas the secondary outcome of interest was the quantification of fall risk. Two independent reviewers (RL and LP) screened all titles, abstracts and full texts for eligibility, and the authors evaluated the studies identified by the searches based on the inclusion and exclusion criteria established.

This review included articles that utilized the following MeSH terms "elderly" AND "vibration" AND "balance". The reference lists for most of the relevant studies were scanned for additional citations. Country, author, affiliated institution, and enrollment period data were extracted and reviewed to identify and exclude duplicate publications using the same cohort. Any disagreement regarding accepting full-text articles was resolved by discussion until consensus was reached.

\section{Study Criteria and Selection}

The inclusion criteria were as follows: (1) published between May 1, 2009 and June 30, 2019; (2) assessed balance or the risk of falls in elderly populations; (3) randomized clinical trials, including crossover and prospective design studies using focused vibration; (4) availability of a full English text; and (5) population age $>60$ years.

The exclusion criteria were as follows: (i) neurological diseases; (ii) treatment with whole-body vibration; (iii) vibratory insoles or footwear; (iv) animal or in vitro studies; and (v) lack of English abstract or English full text.

\section{Data Extraction}

The investigators retrieved all the information from each study. After the application of the eligibility criteria, the included studies were analyzed based on sample demographics, study aims, conflict of interest statements, study durations and follow-up (time and percentage), vibration devices that were used, evaluation times, intervention protocols, and the outcome parameters assessed (clinical and functional).

\section{Methodology Quality Assessment}

The assessment of the quality and risk of bias was done independently by two authors (RL and LP). The quality of each study was assessed using the PEDro scale, ${ }^{23}$ which consists of 11 items that are related to scientific rigor. Items 2 through 11 on the scale contribute to internal validity, and each study is awarded 1 point for each criterion that is not met by the study. The first item relates to external validity and is not included in the final score. To describe the potential for bias for each individual study, the level of evidence presented by each study was assessed according to the Oxford Centre for Evidence-Based Medicine system, which uses 5 levels of evidence, with Level 1 being the highest (eg, systematic reviews and meta-analyses) and Level 5 being the lowest (eg, expert opinion). ${ }^{24}$ These criteria, suggested by Law and MacDermid, have been recommended as being adequate for the evaluation of rehabilitation literature. ${ }^{25}$

\section{Results}

Figure 1 shows the PRISMA flow diagram for a selection of studies. A total of 8 articles $(\mathrm{N}=8)$ satisfied the inclusion criteria and were considered in the review, and detailed information for each study can be found in Tables 1 and 2. Furthermore, in Table 3 the results of the single studies are summarized as mean and SD, only the data concerning balance and/or risk of falling. The PEDro score values and levels of evidence for each of the included studies can be found in Table 4. The studies comprised a total of 635 participants, including 551 females and 84 males. Different protocols applied during each study can be observed in Table 2 .

Most of the included studies ${ }^{26-31}$ featured a control group of similar age, except for $\mathrm{Yu}$ et $\mathrm{al}^{32}$ and Ehsani et $a{ }^{33}$ in which the elderly treatment group was compared with healthy young people. These 2 studies sought to explain how and why vibration conditions could affect balance control in the elderly population compared with young adults. $\mathrm{Yu}$ et $\mathrm{al}^{32}$ attempted to determine agerelated changes under various vibration conditions, especially when vibrations were applied to the tibialis anterior tendon (TAT) and the Achilles tendon (AT), individually or in combination, during 1- and 2-legged stances, and Ehsani et $\mathrm{al}^{33}$ examined the effects of lowfrequency, low-amplitude vibratory stimulation of both Gastrocnemius muscles by including a third group of 


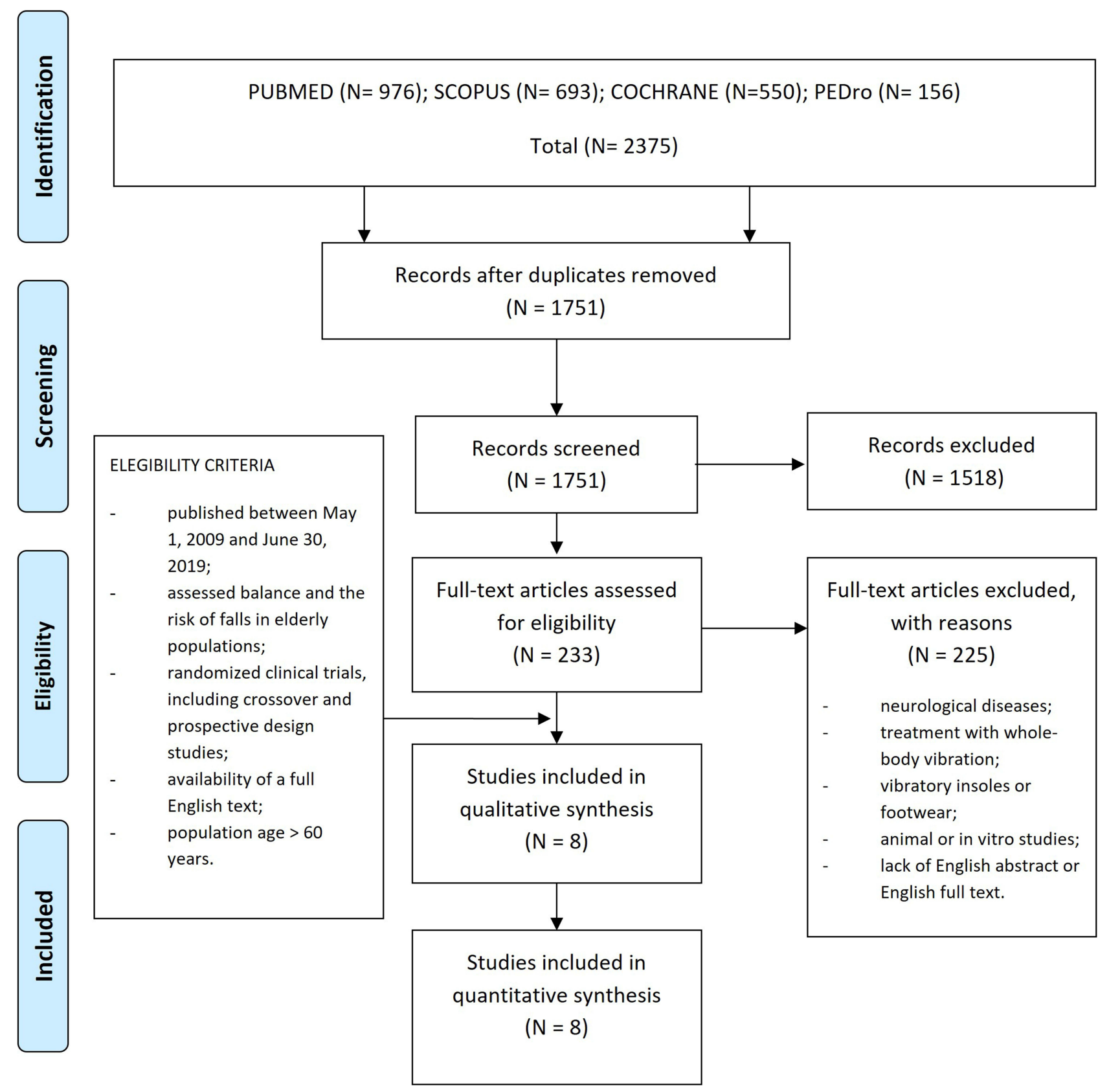

Figure I PRISMA flow-diagram indicating the selection of studies included in the review.

Notes: PRISMA figure adapted from Moher D, Liberati A, Altman D, Tetzlaff J, et al. The PRISMA statement for reporting systematic reviews and meta-analyses of studies that evaluate health care interventions: explanation and elaboration. Journal of Clinical Epidemiology. 2009;62(10). Creative Commons ${ }^{21}$.

elderly people with high fall risk. Ehsani et $\mathrm{al}^{33}$ only used evaluation scales to stratify the sample, based upon the Center for Disease Control and Prevention's STEADI Risk for Falling Assessment questionnaire, ${ }^{34}$ and administered a visual analog pain scale for the lower extremities (VAS-10), ${ }^{35}$ during a 2 -week period prior to the visit and at the time of the visit, and administered the short Falls Efficacy Scale-International (Short FES-I) to assess the fear of falling. ${ }^{36}$
When assessing balance as the primary outcome, 4 studies Bellomo et al, Filippi et al, Wanderley et al, and $\mathrm{Yu}$ et $\mathrm{al}^{26-32}$ used balance platforms to evaluate postural stability by calculating body sway relative to CoP displacement. In contrast, Ehsani et $\mathrm{a}^{33}$ used a wearable motion sensor (a tri-axial gyroscope designed to estimate 3-dimensional ankle and hip angles) to calculate the center of gravity (CoG). Moreover, Wanderley et al, ${ }^{21}$ in addition to assessing balance, assessed performance on the one-leg 


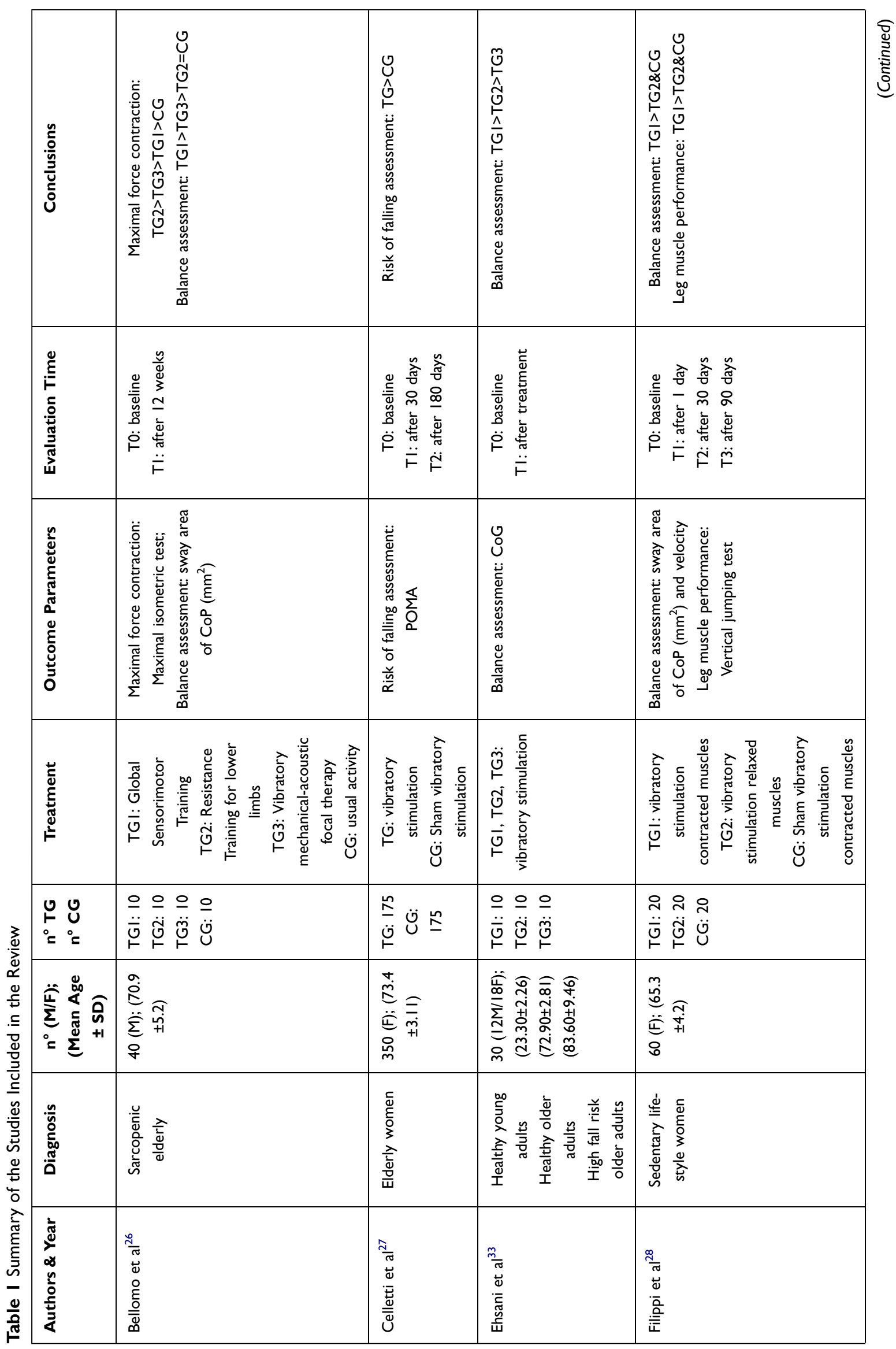




\begin{tabular}{|c|c|c|c|c|c|}
\hline 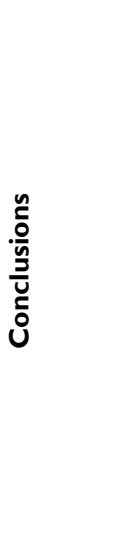 & 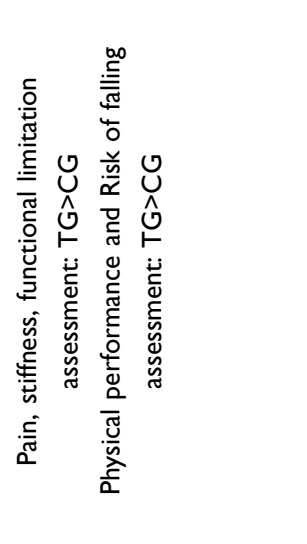 & 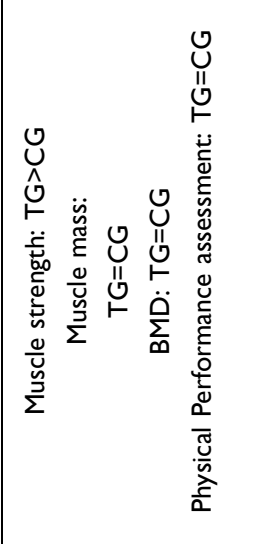 & 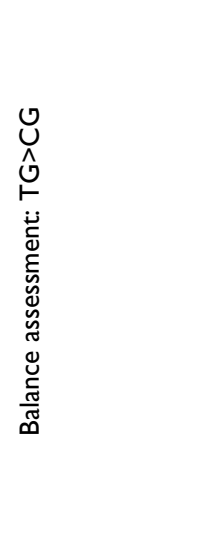 & 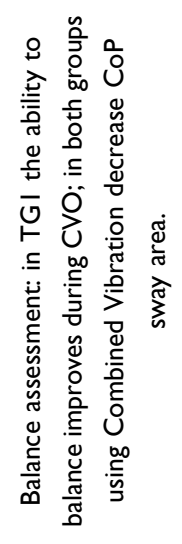 & 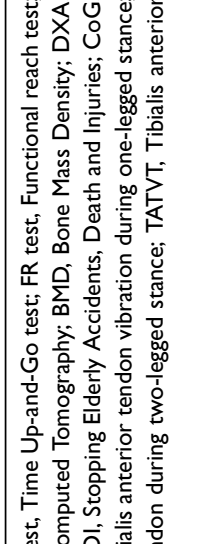 \\
\hline 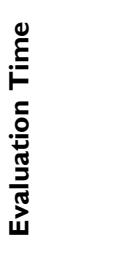 & 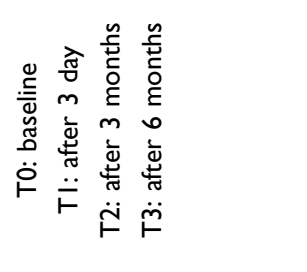 & 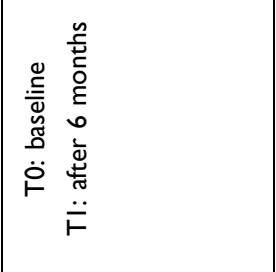 & 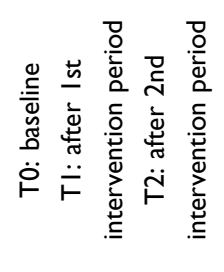 & 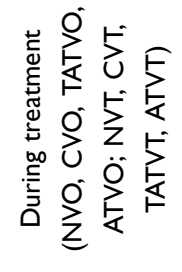 & 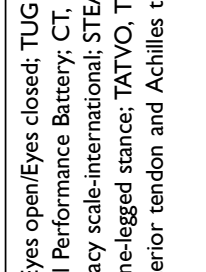 \\
\hline 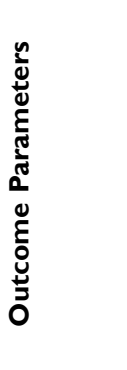 & 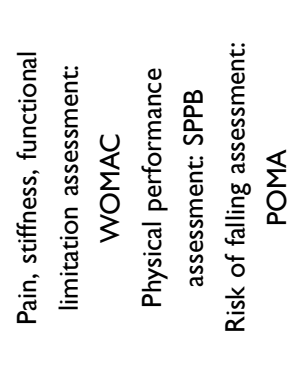 & 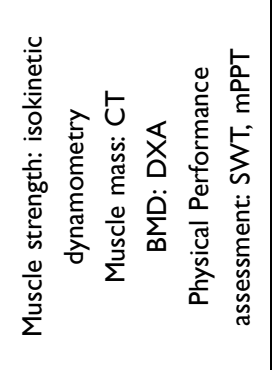 & 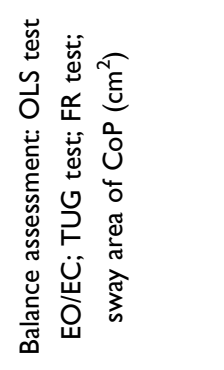 & 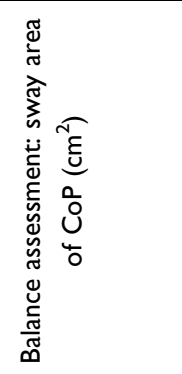 & 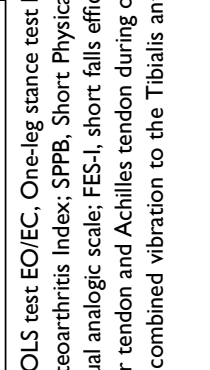 \\
\hline & 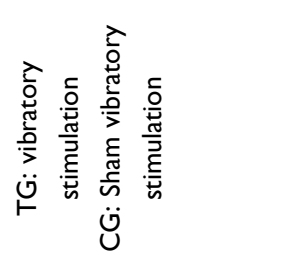 & 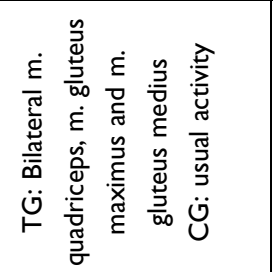 & 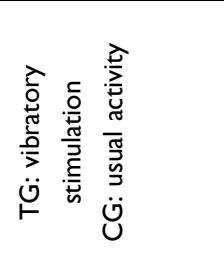 & 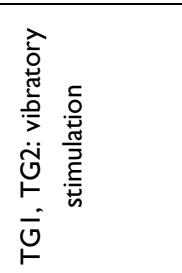 & 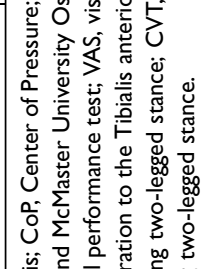 \\
\hline $\begin{array}{ll}0 & 0 \\
0 & 0 \\
\circ & 0 \\
= & 0\end{array}$ & 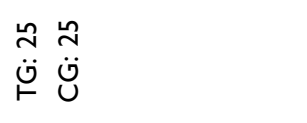 & $\begin{array}{ll}n & \infty \\
\dot{\varphi} & \ddot{ن} \\
\vdash & 0\end{array}$ & 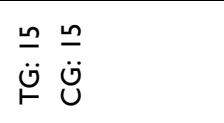 & 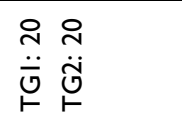 & 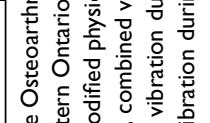 \\
\hline 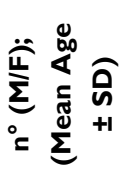 & 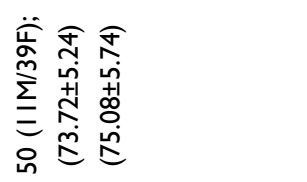 & 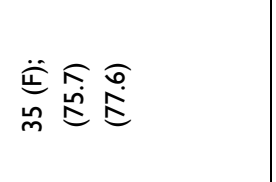 & 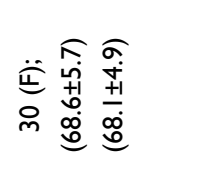 & 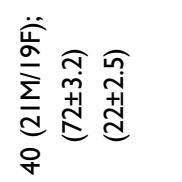 & 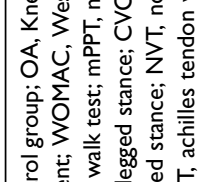 \\
\hline 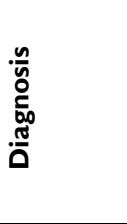 & 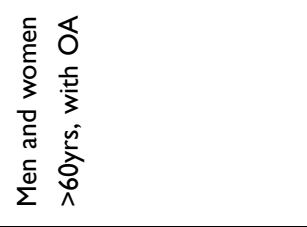 & 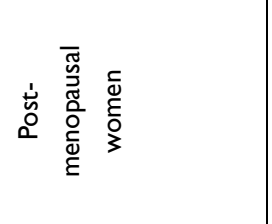 & 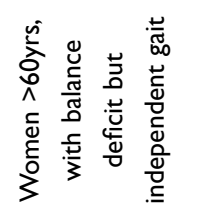 & 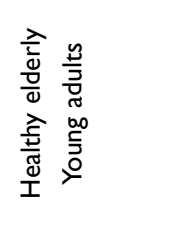 & 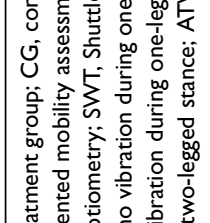 \\
\hline 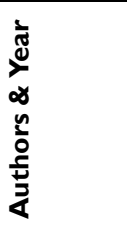 & 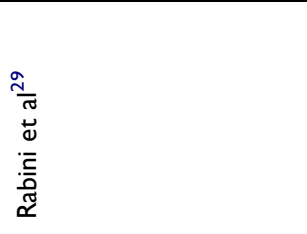 & 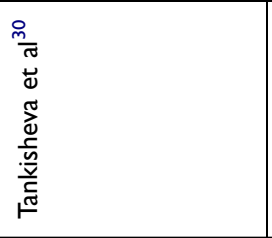 & 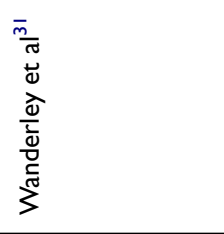 & 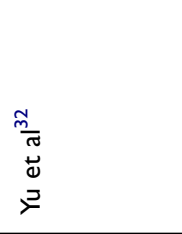 & 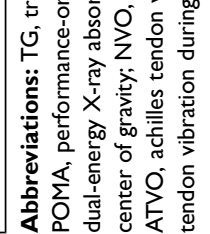 \\
\hline
\end{tabular}




\begin{tabular}{|c|c|c|c|c|c|c|c|c|c|}
\hline 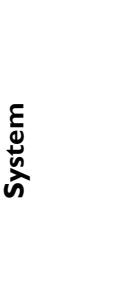 & $\stackrel{\varkappa}{\nu}$ & & 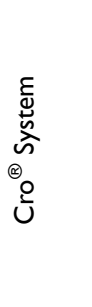 & 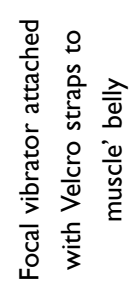 & 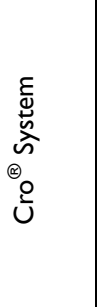 & 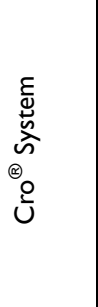 & 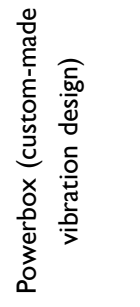 & 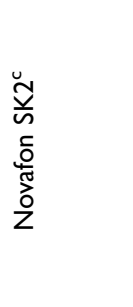 & $\bar{\Xi}$ \\
\hline 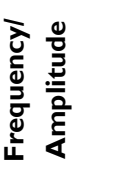 & $\begin{array}{l}\text { N } \\
\text { o } \\
\end{array}$ & & 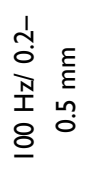 & 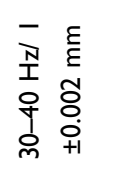 & 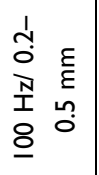 & 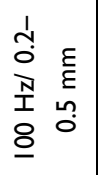 & $\begin{array}{l}\text { N } \\
\text { 忽 } \\
\stackrel{\sim}{N}\end{array}$ & 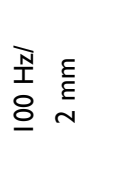 & 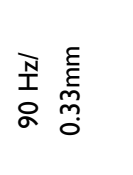 \\
\hline 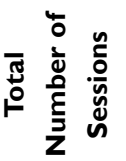 & ণ্ & & $m$ & $\infty$ & $m$ & $m$ & $\stackrel{\text { I }}{\text {. }}$ & $\stackrel{ \pm}{\sim}$ & $\infty$ \\
\hline 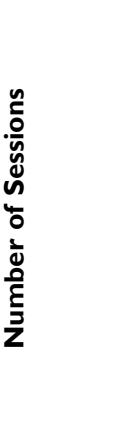 & 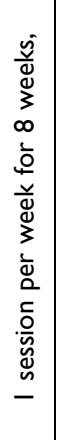 & 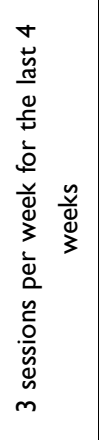 & 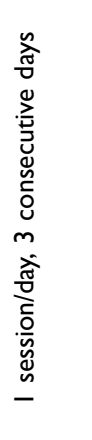 & 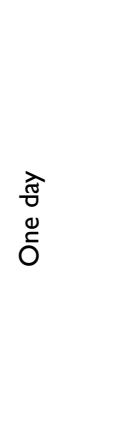 & 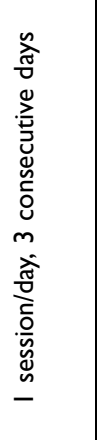 & 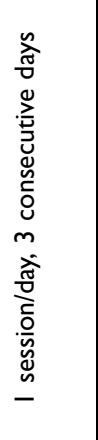 & 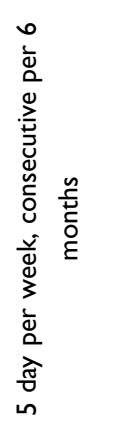 & 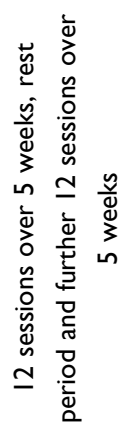 & 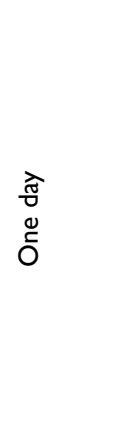 \\
\hline 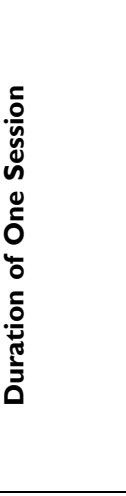 & 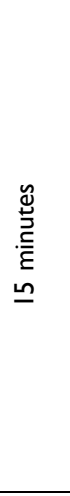 & & 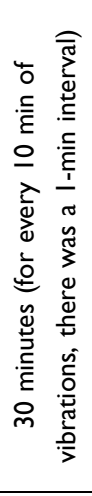 & 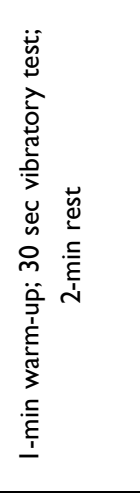 & 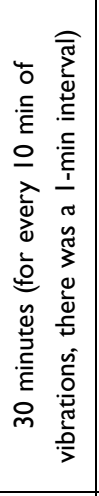 & 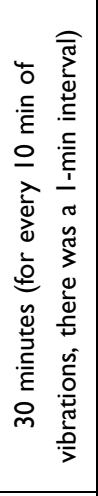 & 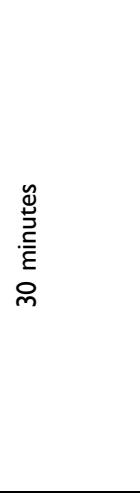 & 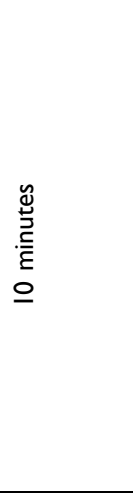 & 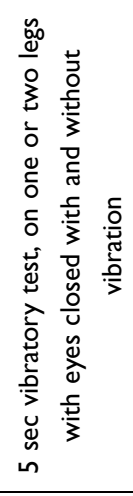 \\
\hline 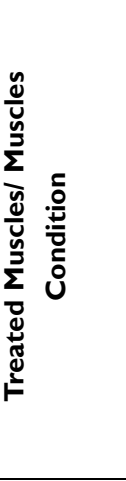 & 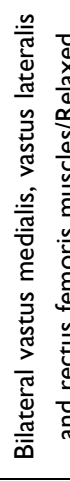 & & 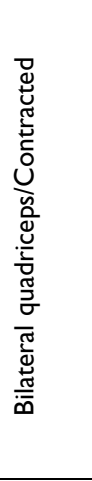 & 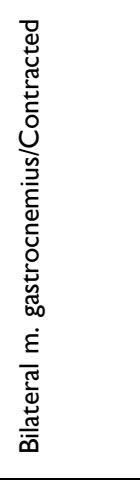 & 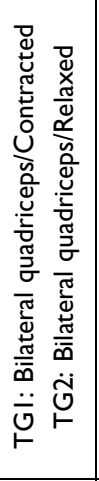 & 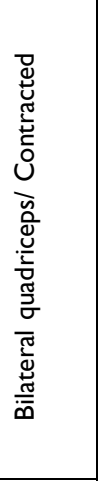 & 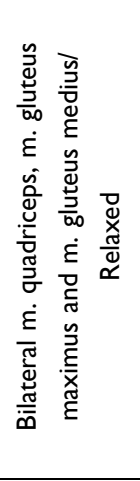 & 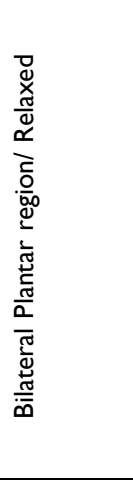 & 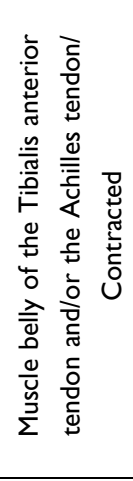 \\
\hline 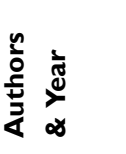 & 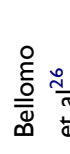 & & 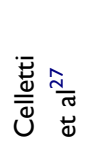 & 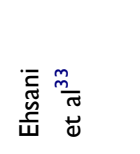 & 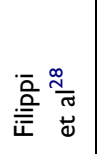 & 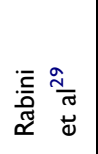 & 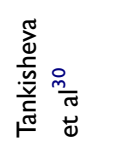 & 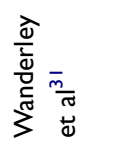 & 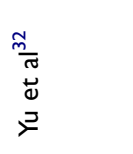 \\
\hline
\end{tabular}


Table 3 The Results Effect of Focused Vibration on Balance and/or Risk of Fall Assessment: Between-Group Differences

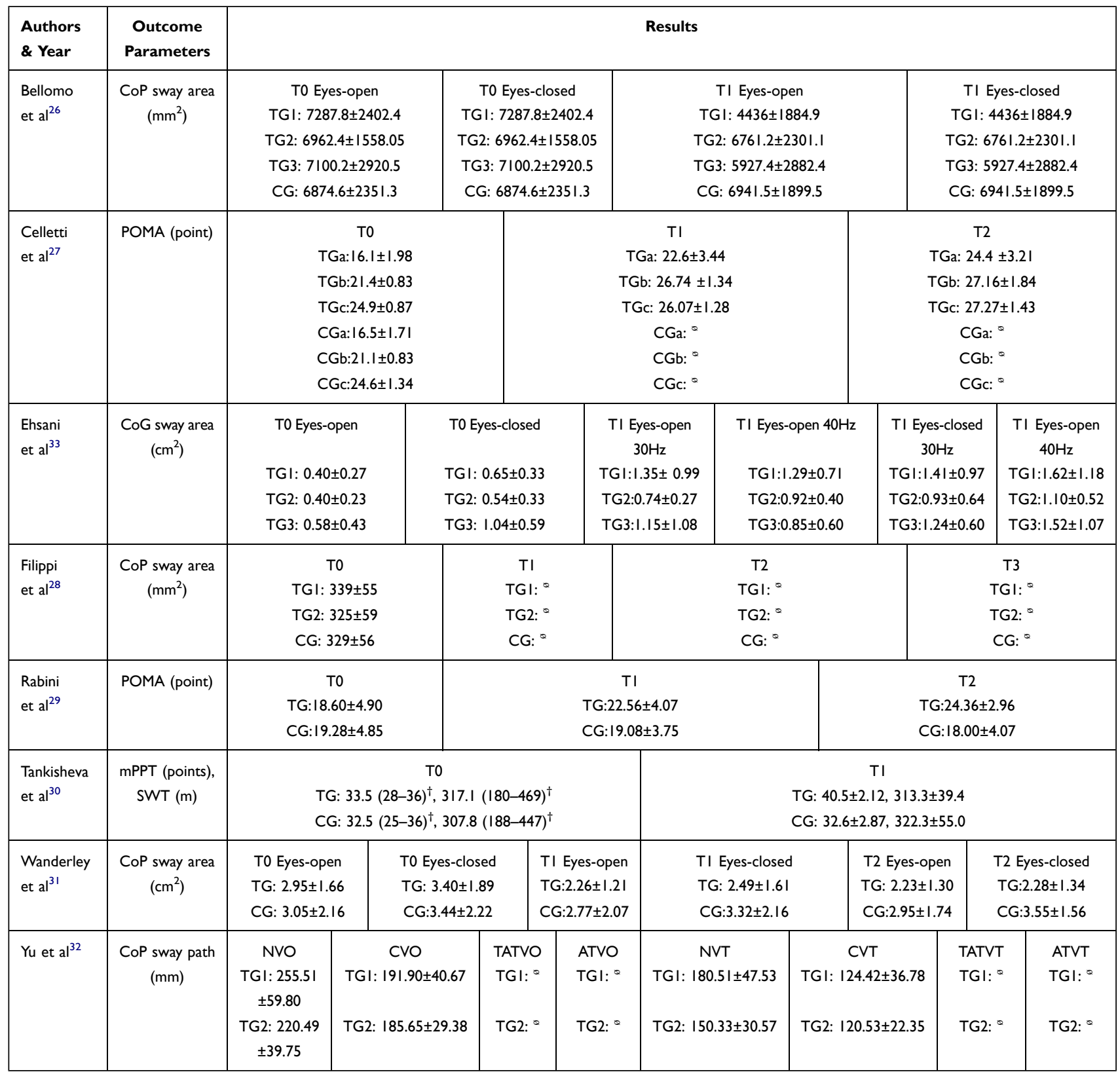

Notes: Values are expressed as mean \pm SD or ranges in parentheses. ${ }^{\top}$ Indicated value not detectable, because just graphical. ${ }^{\dagger}$ Indicate range value (minimum and maximum). Abbreviations: TG, treatment group; CG, control group; a, patient with high risk of falling; b, patient with moderate risk of falling; c, patient with low risk of falling; CoP, Centre of pressure; CoG, Center of gravity; POMA, performance-oriented mobility assessment; SWT, Shuttle walk test; mPPT, modified physical performance test; NVO, No vibration during one-legged stance; CVO, combined vibration to the Tibialis anterior tendon and Achilles tendon during one-legged stance; TATVO, Tibialis anterior tendon vibration during one-legged stance; ATVO, achilles tendon vibration during one-legged stance; NVT, no vibration during two-legged stance; CVT, combined vibration to the Tibialis anterior tendon and Achilles tendon during two-legged stance; TATVT, Tibialis anterior tendon vibration during two-legged stance; ATVT, achilles tendon vibration during two-legged stance.

stance test (OLS test), with open and closed eyes (EO/ EC), ${ }^{37}$ during which the subject was asked to remain in a unipodal position as long as possible, for both legs, up to a maximum of 30". Performance on the Functional Reach test was also assessed, ${ }^{38}$ during which the subject stood with feet shoulder-width apart and arms extended in front of the body parallel to the floor. From this position, the subject displaced his or her body in a forward motion, with the hands open and arms extended as much as possible, without receiving additional support. In addition, Wanderley et $\mathrm{al}^{31}$ used the Timed Up \& Go test ${ }^{39}$ to assess dynamic postural balance, during which the subject was instructed to rise from a chair (with a back but no arms), walk a distance of $3 \mathrm{~m}$, turn $180^{\circ}$, and sit down on the 


\begin{tabular}{|c|c|c|c|c|c|c|c|c|}
\hline 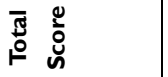 & $\frac{\circ}{7}$ & $\stackrel{\circ}{\gtrless}$ & $\frac{\circ}{f}$ & $\frac{\circ}{6}$ & $\frac{\circ}{\sigma}$ & $\frac{0}{6}$ & $\stackrel{\circ}{ }$ & $\frac{\circ}{7}$ \\
\hline 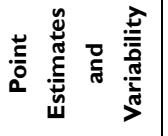 & $\stackrel{\mathscr{\Xi}}{\check{\Xi}}$ & $\stackrel{\check{\varpi}}{\check{\nu}}$ & $\stackrel{\check{\Xi}}{\check{\nu}}$ & $\stackrel{\stackrel{y}{*}}{\tau^{\prime}}$ & 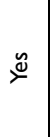 & 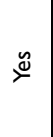 & $\stackrel{\check{\Delta}}{\check{\nu}}$ & 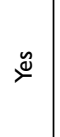 \\
\hline 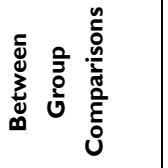 & $\stackrel{\circ}{z}$ & $\stackrel{\mathscr{y}}{\nu}$ & $\stackrel{\check{ٌ}}{\check{\nu}}$ & $\stackrel{\tilde{u}}{\check{\nu}}$ & $\stackrel{\mathscr{y}}{\tau^{y}}$ & 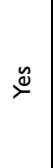 & $\stackrel{\mathscr{ٌ}}{\nearrow}$ & 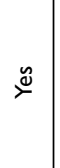 \\
\hline 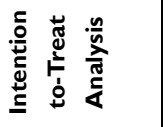 & $\stackrel{\circ}{z}$ & ㅇ & 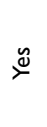 & $\stackrel{\circ}{z}$ & 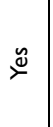 & $\stackrel{0}{z}$ & $\stackrel{\check{ٌ}}{\nearrow}$ & $\stackrel{0}{z}$ \\
\hline 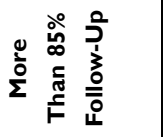 & 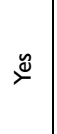 & $\stackrel{\check{\varpi}}{\check{\nu}}$ & $\stackrel{\check{\Xi}}{\approx}$ & 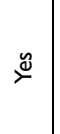 & 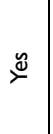 & 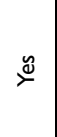 & $\stackrel{\check{ٌ}}{\nearrow}$ & 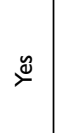 \\
\hline 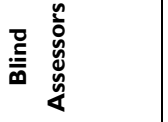 & 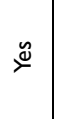 & $\stackrel{\check{\nu}}{\check{\nu}}$ & $\stackrel{0}{z}$ & 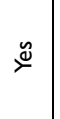 & 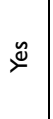 & $\stackrel{\tilde{y}}{\tau}$ & $\stackrel{\check{ٌ}}{\sim}$ & $\stackrel{0}{z}$ \\
\hline 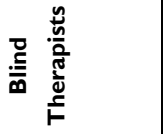 & $\stackrel{\circ}{z}$ & ż & $\stackrel{\circ}{z}$ & $\stackrel{\circ}{z}$ & $\stackrel{\circ}{z}$ & $\stackrel{\circ}{z}$ & $\stackrel{\circ}{z}$ & $\stackrel{\circ}{z}$ \\
\hline 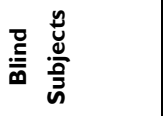 & $\stackrel{\circ}{z}$ & zo & $\stackrel{\circ}{z}$ & $\stackrel{\circ}{z}$ & 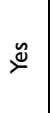 & $\stackrel{\circ}{z}$ & $\stackrel{\circ}{z}$ & $\stackrel{0}{z}$ \\
\hline 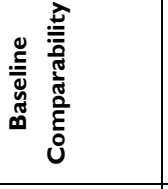 & $\stackrel{\circ}{z}$ & $\stackrel{\mathscr{\Xi}}{\check{\nu}}$ & $\stackrel{0}{z}$ & $\stackrel{\mathscr{\Xi}}{\longleftarrow}$ & 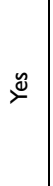 & 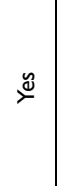 & $\stackrel{\check{ٌ}}{\check{\nu}}$ & $\stackrel{\Xi}{\nu}$ \\
\hline 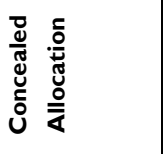 & $\stackrel{o}{z}$ & $\stackrel{\mathscr{E}}{\check{\tilde{E}}}$ & zo & $\stackrel{0}{z}$ & 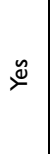 & $\stackrel{0}{z}$ & $\stackrel{\circ}{z}$ & $\stackrel{0}{z}$ \\
\hline 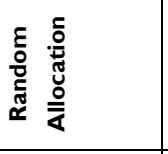 & 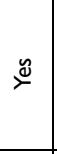 & $\stackrel{\mathscr{g}}{\check{\nu}}$ & $\stackrel{\circ}{z}$ & $\stackrel{\mathscr{\Xi}}{\longleftarrow}$ & 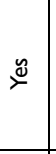 & 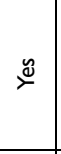 & $\stackrel{\check{ٌ}}{\check{0}}$ & ㅇ \\
\hline 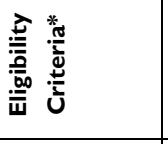 & $\stackrel{\circ}{z}$ & \& & 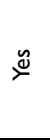 & $\check{\iota}^{\mathscr{y}}$ & 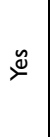 & 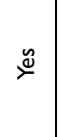 & 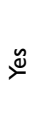 & $\stackrel{\check{\Perp}}{\check{\nu}}$ \\
\hline 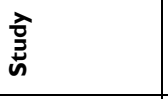 & $\begin{array}{l}u \\
\stackrel{c}{\alpha}\end{array}$ & $\begin{array}{l}u \\
\underline{\alpha}\end{array}$ & $\tilde{u}$ & $\underset{\underline{\alpha}}{U}$ & $\begin{array}{l}U \\
\check{c}\end{array}$ & $\underset{\underline{x}}{u}$ & $\underset{\propto}{u}$ & $\tilde{u}$ \\
\hline 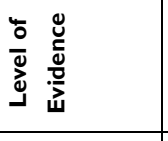 & $\equiv$ & $=$ & $\equiv$ & $=$ & $=$ & $=$ & $=$ & $\equiv$ \\
\hline 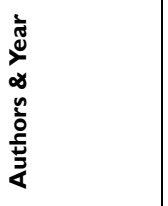 & 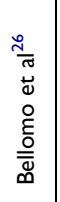 & 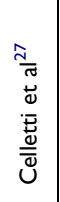 & 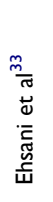 & 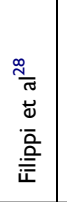 & 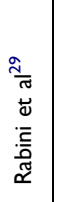 & 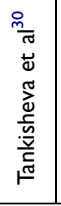 & 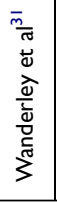 & 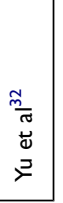 \\
\hline
\end{tabular}


chair again (a time longer than 13.5 seconds indicates an increased risk of falling among elderly subjects). Bellomo et al and Tankisheva et $\mathrm{al}^{26-30}$ measured dynamic postural balance, respectively, using gait analysis (on a podobarographic platform, 4 meters long) and the shuttle walk test (SWT), ${ }^{40}$ which is a standardized, incremental, submaximal field-walking test.

To assess balance, gait and the risk of falling, the included studies ${ }^{27-29}$ used rating scales, such as the Performance-Oriented Mobility Assessment (POMA) scale. ${ }^{41}$ Rabini et al $^{29}$ also evaluated physical performance using the short physical performance battery (SPPB) ${ }^{42}$ and Western Ontario and McMaster Universities Arthritis Index (WOMAC) scores, ${ }^{43}$ to quantify pain and functional limitations of the knee.

To evaluate leg muscle performance, Filippi et $\mathrm{al}^{28}$ used the vertical jumping test, ${ }^{44}$ quantified using a device that allows the direct measurement of jump time and vertical body displacement during the jump, whereas leg power was indirectly computed, considering body weight and wire displacement. Bellomo et $\mathrm{al}^{26}$ evaluated the maximum isometric strength of each subject using a leg extension machine. After adjusting the position of each subject, to achieve a $90^{\circ}$ knee angle and a $90^{\circ}$ hip flexion angle, the subject was asked to perform a maximum contraction for 5 seconds. The measurement was repeated 3 times, with a 2-minute rest period between each test. The best result among the 3 tests was the maximal isometric force (ISOmax).

A total of 6 different vibration devices were used among the 8 included studies, each of which was associated with different FV frequency and amplitude characteristics and different treatment protocols. Celletti et al, Filippi et al and Rabini et $\mathrm{al}^{27-29}$ all used the CroSystem device, consisting of an electromechanical transducer, mechanical support that was rigidly attached to the floor, and an electronic control device. A mechanical arm allowed the transducer to be placed on a muscle belly, using a specific "repeated Muscle Vibration" (rMV) protocol that generated a sinusoidal displacement at $100 \mathrm{~Hz}$, $0.2-0.5 \mathrm{~mm}$ peak to peak. In contrast, Bellomo et $\mathrm{al}^{26}$ used a Vibration Sound System ${ }^{\circledR}$ (Vissman s.r.l., Rome, Italy) device that administered focused mechano-acoustic vibrations using a turbine, with a flow rate of $35 \mathrm{~m}^{3} /$ hour, which was able to generate airwaves with a pressure of up to 250 mbar, and of a flow modulator, capable of vibrating air with a pressure of up to 630 mbar and a frequency of up to
$980 \mathrm{~Hz}$ (a frequency within $300 \mathrm{~Hz}$ is recommended), producing mechano-acoustic waves.

\section{Discussion}

The purpose of this review was to assess the effects of FV on postural control and reductions in the risk of falling among the elderly population. This review, despite the few studies included, can demonstrate the positive effects of FV stimulus on balance, despite 1 study $^{30}$ that found that FV stimulation had no effects on the physical performance of postmenopausal women.

However, discrepancies in the vibratory parameters used were identified among the included studies. According to the experience reported by Filippi et al, ${ }^{28}$ short applications and low frequencies are less effective than other protocols. Based on the studies that showed improvements in motor performance and balance, the FV stimulus must selectively stimulate the type Ia futsal afferents, using frequencies at $100 \mathrm{~Hz}$ and amplitudes from 0.2 to $0.5 \mathrm{~mm} .^{45}$ These parameters were used by 5 of the included studies, ${ }^{27-32}$ whereas type Ib and II afferent fibers can be recruited when larger amplitudes are used. ${ }^{46}$ Several receptor structures have been reported to be sensitive to vibratory stimuli. Cutaneous receptors, such as Pacini, Merkel, Meissner, and Ruffini receptors, can be activated by FV at various frequencies, and the Golgi tendon organs are sensitive to the vibration of $\mathrm{Ib}$ afferents. $^{15}$

However, various studies have shown that the most sensitive receptor structures are the neuromuscular spindles, which consist of primary (Ia), which are the most sensitive, and secondary (II) fiber afferents. The type Ia afferents are activated during small FV amplitudes, from 0.2 to $0.5 \mathrm{~mm}$, and type I afferents respond to frequencies up to $120 \mathrm{~Hz}$, proportionally, one by one, whereas the type $\mathrm{Ib}$ and II afferents respond to greater amplitudes, and the type II afferents are recruited from 20 to $60 \mathrm{~Hz}^{47}$ The responses of type Ia afferents are dependent on whether the muscle is stretched, relaxed, or contracted, ${ }^{48}$ and type Ia afferents are more responsive to FV when the muscle is stretched and during voluntary isometric contractions. ${ }^{27-29}$ Moreover, muscle spindle shots induced by FV excite not only motor neurons but also interneurons in the spinal cord, which reciprocally inhibit the motor neurons innervating antagonistic muscles. ${ }^{49}$

According to recent literature, the vibration-induced activation of spindles can induce the long-term reorganization of the central nervous system, if the primary spinal 
afferent activity is strong and prolonged. ${ }^{47}$ Long-term effects were observed only when using high-intensity vibrations $(100 \mathrm{~Hz}){ }^{28}$ Long-term effects were not assessable for all included studies, as only 3 studies reported long-term follow-up (3 months). ${ }^{27-29}$ Among these studies, the $100-\mathrm{Hz}$ vibratory stimulus-induced stability improvements, which generally persisted after 3 months. In contrast, Bellomo et $\mathrm{al}^{26}$ used $300-\mathrm{Hz}$ stimulations with high-intensity frequencies and observed improvements; however, this study did not include long-term follow-up. Unusual parameters were reported for the studies performed by Tankisheva et al and Ehsani et $\mathrm{al}^{30-33}$ who applied a frequency of $30-45 \mathrm{~Hz}$. Tankisheva et al did not report any improvements in muscle strength or physical performance assessment, ${ }^{30}$ whereas Ehsani et al detected improvements in balance after 1 month of followup. ${ }^{33}$ Given the discordant results between the only 2 studies that used low-frequency FV parameters (from 25 to $45 \mathrm{~Hz}$ ) Tankisheva et al and Ehsani et al, ${ }^{30-33}$ we cannot conclude that low-frequency $\mathrm{FV}$ has positive effects. However, the limitations and poor quality of some of the included studies must be considered: in fact, this review included 3 articles with a PEDro score of 4 (fair quality), including 2 non-randomized articles (cross-sectional and clinical study $)^{32,33}$ and 1 randomized, clinical trial. ${ }^{26}$ In general, FV appears to have positive effects on the postural stability of the elderly.

Postural control represents a complex motor skill, derived from the interactions among multiple sensorimotor processes, including primary contributions from the auditory, visual, and vestibular systems. ${ }^{50}$ In general, healthy adults rely on somatosensory (70\%), visual $(10 \%)$, and vestibular (20\%) information; however, when they stand on an unstable surface, they increase sensory weighting to prioritize vestibular and visual information and decrease their dependence on surface somatosensory inputs, for improved postural orientation. ${ }^{51}$ Among the elderly population, these multiple sensory inputs may be insufficient or difficult to integrate; thus, poor vision, low handgrip strength, walking speed declines, the use of walking aids, drug use, and depression are recognized to be risk factors for falls among the elderly population. ${ }^{9}$

A person who is standing in an upright position can maintain balance thanks to small but continuous oscillations that are intended to counterbalance gravitational forces. The $\mathrm{CoG}$ represents the vertical projection onto the ground and the center of mass (CoM) of the body; $\mathrm{CoP}$ and $\mathrm{CoG}$ only coincide perfectly under static conditions when the moment of the muscular ankle articulation force equals the gravitational force. ${ }^{52}$ Balance is only achieved when these 2 vectors are aligned along the vertical axis of the subject.

Therefore, 2 mechanical models can be used to describe postural dynamics: hip strategy, which depends on the mobilization of the CoG, and ankle strategy, which depends on the mobilization of the CoP. The ankle strategy, during which the body moves by using the ankle as a flexible, inverted pendulum, is appropriate for maintaining balance in response to small amounts of sway when standing on a firm surface. The hip strategy, during which the body exerts torque at the hips to quickly move the $\mathrm{CoM}$, is used when persons stand on narrow or compliant surfaces that do not allow adequate ankle torque or when CoM must be moved quickly. ${ }^{53}$ The purposes of these strategies are to obtain optimal vertical alignment, allowing the subject to maintain $\mathrm{CoG}$ within a support polygon. Accelerometers are used to evaluate CoM dynamic performance thank you to the sensitivity of these devices to small changes in postural control systems, and it allows us to identify early changes in spatiotemporal gait parameters. ${ }^{54,55}$ Furthermore, Leirós-Rodríguez et al. They have shown that the accelerometric gait assessment can detect alterations in the balance during aging, by detecting differences in women between 51 and 80 years. Their results indicate that during the aging process the velocity and acceleration are reduced and thus the reduction in speed makes the subject more susceptible to falls. ${ }^{56}$ This review included 3 studies $^{31-33}$ that focused on FV stimulation of the ankle and plantar muscles, whereas 5 studies $^{26-30}$ utilized the stimulation of the quadriceps muscles, to improve balance in an elderly population. The quadriceps muscles are extremely important for ADL, including standing up, sitting down, stair-climbing, and gait. Furthermore, several studies have investigated the effects of FV applications on quadriceps muscle strength, and the prolonged vibratory stimulation of the quadriceps femoris, both at 80 and $300 \mathrm{~Hz}$, resulted in increased muscle strength, which persisted at follow-up, suggesting an underlying plastic process. ${ }^{57,58}$

\section{Conclusion}

This review suggests that FV can be an effective training method that can decrease the risk of falls, which is a major cause of dependency among older populations. The use of FV reduces the speed and displacement of $\mathrm{CoP}$ and improves the results on various tests of physical 
performance, which increases balance. FV may be used as an alternative to classical methods used for strength and proprioceptive training, such as whole-body vibration training. Moreover, FV does not require an active contribution from the subject and can directly target a specific muscle group, making it easier to implement for improved balance and reduced fall risk among the elderly population.

However, future studies should use strong methodological quality control, including the use of a control group with similar initial characteristics, provide for long-term follow-up, and be able to identify a single adequate and reproducible protocol, detailing all necessary parameters and times.

\section{Disclosure}

The authors report no conflicts of interest in this work.

\section{References}

1. Liotta G, Canhao H, Cenko F, et al. Active ageing in Europe: adding healthy life to years. Front Med (Lausanne). 2018;5:123. doi:10. 3389/fmed.2018.00123

2. Pietrangelo T, Mancinelli R, Toniolo L, et al. Effects of local vibrations on skeletal muscle trophism in elderly people: mechanical, cellular, and molecular events. Int J Mol Med. 2009;24(4):503-512. doi:10.3892/ijmm_00000259

3. Fulle S, Protasi $\bar{F}$, Di Tano G, et al. The contribution of reactive oxygen species to sarcopenia and muscle ageing. Exp Gerontol. 2004;39(1):17-24. doi:10.1016/j.exger.2003.09.012

4. Fulle S, Belia S, Di Tano G. Sarcopenia is more than a muscular deficit. Arch Ital Biol. 2005;143(3):229-234.

5. Brown WF, Strong MJ, Snow R. Methods for estimating numbers of motor units in biceps-brachialis muscles and losses of motor units with aging. Muscle Nerve. 1988;11(5):423-432. doi:10.1002/mus. 880110503

6. Leirós-Rodríguez R, Romo-Pérez V, García-Soidán JL, García-Liñeira J. Percentiles and reference values for the Accelerometric assessment of static balance in women aged 50-80 years. Sensors. 2020;20(3):940. doi:10.3390/s20030940

7. Beers MH. The Merck Manual of Health \& Aging. Random House Digital, Inc; 2005.

8. Rubenstein LZ. Falls in older people: epidemiology, risk factors and strategies for prevention. Age Ageing. 2006;35(Suppl 2):ii37-ii41. doi:10.1093/ageing/afl084

9. Collard RM, Boter H, Schoevers RA, Oude Voshaar RC. Prevalence of frailty in community-dwelling older persons: a systematic review. $J$ Am Geriatr Soc. 2012;60(8):1487-1492. doi:10.1111/j.15325415.2012.04054.x

10. Intiso D, Di Rienzo F, Russo M, et al. Rehabilitation strategy in the elderly. $J$ Nephrol. 2012;25(Suppl 19):S90-5. doi:10.5301/jn.500 0138

11. Thomas E, Battaglia G, Patti A, et al. Physical activity programs for balance and fall prevention in elderly: a systematic review. Medicine (Baltimore). 2019;98(27):e16218. doi:10.1097/MD.0000000000016 218

12. Rees SS, Murphy AJ, Watsford ML. Effects of whole-body vibration on postural steadiness in an older population. $J$ Sci Med Sport. 2009;12(4):440-444. doi:10.1016/j.jsams.2008.02.002
13. Šarabon N, Kozinc Ž, Löfler S, Hofer C. Resistance exercise, electrical muscle stimulation, and whole-body vibration in older adults: systematic review and meta-analysis of randomized controlled trials. J Clin Med. 2020;9(9):2902. doi:10.3390/jcm9092902

14. Kasturi GC, Adler RA. Osteoporosis: nonpharmacologic management. PM R. 2011;3(6):562-572. doi:10.1016/j.pmrj.2010.12.014

15. Griffin MJ. Minimum health and safety requirements for workers exposed to hand-transmitted vibration and whole-body vibration in the European Union; a review. Occup Environ Med. 2004;61 (5):387-397. doi:10.1136/oem.2002.006304

16. Souron R, Besson T, Millet GY, Lapole T. Acute and chronic neuromuscular adaptations to local vibration training. Eur J Appl Physiol. 2017;117(10):1939-1964. doi:10.1007/s00421-017-3688-8

17. Aboutorabi A, Arazpour M, Bahramizadeh M, Farahmand F, Fadayevatan R. Effect of vibration on postural control and gait of elderly subjects: a systematic review. Aging Clin Exp Res. 2018;30 (7):713-726. doi:10.1007/s40520-017-0831-7

18. Kavounoudias A, Roll R, Roll JP. Foot sole and ankle muscle inputs contribute jointly to human erect posture regulation. $J$ Physiol. 2001;532(3):869-878. doi:10.1111/j.1469-7793.2001.0869e.x

19. Nakagawa HB, Ferraresi JR, Prata MG, Scheicher ME. Postural balance and functional Independence of elderly people according to gender and age: cross-sectional study. Sao Paulo Med J. 2017;135 (3):260-265. doi:10.1590/1516-3180.2016.0325280217

20. Kumar A, Delbaere K, Zijlstra GAR, et al. Exercise for reducing fear of falling in older people living in the community: Cochrane systematic review and meta-analysis. Age Ageing. 2016;45(3):345-352. doi:10.1093/ageing/afw036

21. Moher D, Liberati A, Tetzlaff J, Altman DG; The PRISMA Group. Preferred reporting items for systematic reviews and meta-analyses: the PRISMA statement. PLoS Med. 2009;6(7):e1000097. doi:10.13 71/journal.pmed.1000097

22. van Loveren C, Aartman IH. [The PICO (patient-interventioncomparison-outcome) question]. Ned Tijdschr Tandheelkd. 2007;114 (4):172-178. Dutch.

23. Maher CG, Sherrington C, Herbert RD, Moseley AM, Elkins M. Reliability of the PEDro scale for rating quality of randomized controlled trials. Phys Ther. 2003;83(8):713-721. doi:10.1093/ptj/ 83.8.713

24. Oxford Centre for Evidence-Based Medicine Levels of Evidence Working Group. The Oxford 2011 levels of evidence; 2016. Oxford, England: Oxford Centre for EvidenceBased Medicine. Available from: http://www.cebm.net/index.aspx?o55653. Accessed November 9, 2021.

25. Law MC, MacDermid J. Evidence-Based Rehabilitation: A Guide to Practice. 3rd ed. Thorofare, NJ: Slack; 2014.

26. Bellomo RG, Iodice P, Maffulli T, et al. Muscle strength and balance training in sarcopenic elderly: a pilot study with randomized controlled trial. Eur J Inflamm. 2013;11(1):193-201. doi:10.1177/ 1721727X1301100118

27. Celletti C, Fattorini L, Camerota F, et al. Focal muscle vibration as a possible intervention to prevent falls in elderly women: a pragmatic randomized controlled trial. Aging Clin Exp Res. 2015;27 (6):857-863. doi:10.1007/s40520-015-0356-X

28. Filippi GM, Brunetti O, Botti FM, et al. Improvement of stance control and muscle performance induced by focal muscle vibration in young-elderly women: a randomized controlled trial. Arch Phys Med Rehabil. 2009;90(12):2019-2025. doi:10.1016/j.apmr.2009.08.139

29. Rabini A, De Sire A, Marzetti E, et al. Effects of focal muscle vibration on physical functioning in patients with knee osteoarthritis: a randomized controlled trial. Eur J Phys Rehabil Med. 2015;51(5):513-520.

30. Tankisheva E, Bogaerts A, Boonen S, et al. Effects of a six-month local vibration training on bone density, muscle strength, muscle mass, and physical performance in postmenopausal women. J Strength Cond Res. 2015;29(9):2613-2622. doi:10.1519/JSC.00 00000000000895 
31. Wanderley FS, Alburquerque-Sendín F, Parizotto NA, Rebelatto JR. Effect of plantar vibration stimuli on the balance of older women: a randomized controlled trial. Arch Phys Med Rehabil. 2011;92 (2):199-206. doi:10.1016/j.apmr.2010.10.014

32. Yu M, Piao YJ, Kim SH, Kim DW, Kim NG. Effects of tendon vibration during one-legged and two-legged stance in elderly individuals. Int J Precis Eng Manufactur. 2010;11(6):969-977. doi:10.1007/s12541-010-0117-x

33. Ehsani H, Mohler J, Marlinski V, Rashedi E, Toosizadeh N. The influence of mechanical vibration on local and central balance control. J Biomech. 2018;71:59-66. doi:10.1016/j.jbiomech.2018.01.027

34. Rubenstein LZ, Vivrette R, Harker JO, Stevens JA, Kramer BJ. Validating an evidence-based, self-rated fall risk questionnaire (FRQ) for older adults. $J$ Safety Res. 2011;42(6):493-499. doi:10.1016/j.jsr.2011.08.006

35. Langley GB, Sheppeard H. The visual analog scale - its use in pain measurement. Rheumatol Int. 1985;5(4):145-148. doi:10.1007/ BF00541514

36. Kempen GI, Yardley L, Van Haastregt JC, et al. The Short FES-I: a shortened version of the falls efficacy scale-international to assess fear of falling. Age Ageing. 2008;37(1):45-50. doi:10.1093/ageing/afm157

37. Gustafson AS, Noaksson L, Kronhed AC, Möller M, Möller C. Changes in balance performance in physically active elderly people aged 73-80. Scand J Rehabil Med. 2000;32(4):168-172. doi:10.1080/ 003655000750060913

38. Duncan PW, Weiner DK, Chandler J, Studenski S. Functional reach: a new clinical measure of balance. J Gerontol. 1990;45(6):M192-7. doi:10.1093/geronj/45.6.M192

39. Podsiadlo D, Richardson S. The timed "Up \& Go": a test of basic functional mobility for frail elderly persons. $\mathrm{J}$ Am Geriatr Soc. 1991;39(2):142-148. doi:10.1111/j.1532-5415.1991.tb01616.x

40. Singh SJ, Morgan MD, Scott S, Walters D, Hardman AE. Development of a shuttle walking test of disability in patients with chronic airways obstruction. Thorax. 1992;47(12):1019-1024. doi:10.1136/thx.47.12.1019

41. Tinetti ME. Performance oriented assessment of mobility problems in elderly patients. J Am Geriatr Soc. 1986;34(2):119-126. doi:10.1111/ j.1532-5415.1986.tb05480.x

42. Guralnik JM, Simonsick EM, Ferrucci L, et al. A short physical performance battery assessing lower extremity function: association with self-reported disability and prediction of mortality and nursing home admission. J Gerontol. 1994;49(2):M85-94. doi:10.1093/geronj/49.2. M85

43. Ornetti P, Dougados M, Paternotte S, Logeart I, Gossec L. Validation of a numerical rating scale to assess functional impairment in hip and knee osteoarthritis: comparison with the WOMAC function scale. Ann Rheum Dis. 2011;70(5):740-746. doi:10.1136/ard.2010.135483

44. Sáez Sáez De Villarreal E, Requena B, Arampatzi F, Salonikidis K. Effect of plyometric training on chair-rise, jumping and sprinting performance in three age groups of women. J Sports Med Phys Fitness. 2010;50(2):166-173.
45. Vedel JP, Roll JP. Response to pressure and vibration of slowly adapting cutaneous mechanoreceptors in the human foot. Neurosci Lett. 1982;34(3):289-294. doi:10.1016/0304-3940(82)90190-2

46. Ribot-Ciscar E, Vedel JP, Roll JP. Vibration sensitivity of slowly and rapidly adapting cutaneous mechanoreceptors in the human foot and leg. Neurosci Lett. 1989;104(1-2):130-135. doi:10.1016/03043940(89)90342-X

47. Murillo N, Valls-Sole J, Vidal J, et al. Focal vibration in neurorehabilitation. Eur J Phys Rehabil Med. 2014;50(2):231-242.

48. Souron R, Besson T, Lapole T, Millet GY. Neural adaptations in quadriceps muscle after 4 weeks of local vibration training in young versus older subjects. Appl Physiol Nutr Metab. 2018;43 (5):427-436. doi:10.1139/apnm-2017-0612

49. Brunetti O, Filippi GM, Lorenzini M, et al. Improvement of posture stability by vibratory stimulation following anterior cruciate ligament reconstruction. Knee Surg Sports Traumatol Arthrosc. 2006;14 (11):1180-1187. doi:10.1007/s00167-006-0101-2

50. Thomas E, Martines F, Bianco A, et al. Decreased postural control in people with moderate hearing loss. Medicine (Baltimore). 2018;97 (14):e0244. doi:10.1097/MD.0000000000010244

51. Horak FB. Postural orientation and equilibrium: what do we need to know about neural control of balance to prevent falls? Age Ageing. 2006;35(Suppl 2):ii7-ii11. doi:10.1093/ageing/afl077

52. Gagey B, Bourdeaux O, Gagey PM. From the center of pressure to the center of gravity, a new algorithm for a step forward in stabilometry. Man Ther Posturol Rehabil J. 2015;13:1-5.

53. Horak FB, Kuo A. Postural adaptation for altered environments, tasks and intentions. In: Biomechanics and Neuronal Control of Posture and Movement. New York: Springer; 2000:267-281.

54. Leirós-Rodríguez R, Romo-Pérez V, García-Soidán JL. Validity and reliability of a tool for accelerometric assessment of static balance in women. Eur J Physiother. 2017;19:243-248. doi:10.1080/21679169. 2017.1347707

55. Leirós-Rodríguez R, Arce ME, Souto-Gestal A, García-Soidán JL. Identificación de puntos de referencia anatómicos para la valoración del equilibrio mediante dispositivos cinemáticos. Fisioterapia. 2015;37(5):223-229. doi:10.1016/j.ft.2014.10.006

56. Leirós-Rodríguez R, Romo-Pérez V, García-Soidán JL, SotoRodríguez A. Identification of body balance deterioration of gait in women using accelerometers. Sustainability. 2020;12(3):1222. doi:10.3390/su12031222

57. Hurley MV, Rees J, Newham DJ. Quadriceps function, proprioceptive acuity and functional performance in healthy young, middle-aged and elderly subjects. Age Ageing. 1998;27(1):55-62. doi:10.1093/ ageing/27.1.55

58. Feltroni L, Monteleone S, Petrucci L, et al. Potentiation of muscle strength by focal vibratory stimulation on quadriceps femoris. G Ital Med Lav Ergon. 2018;40(2):90-96.
Clinical Interventions in Aging

\section{Publish your work in this journal}

Clinical Interventions in Aging is an international, peer-reviewed journal focusing on evidence-based reports on the value or lack thereof of treatments intended to prevent or delay the onset of maladaptive correlates of aging in human beings. This journal is indexed on PubMed Central, MedLine, CAS, Scopus and the Elsevier
Bibliographic databases. The manuscript management system is completely online and includes a very quick and fair peer-review system, which is all easy to use. Visit http://www.dovepress.com/ testimonials.php to read real quotes from published authors. 